\title{
Assessment of Scleral Contour Changes in High Myopia by Optical Coherence Tomography
}

\author{
Yong Kyun Shin ${ }^{1}$, Se Woong Kang ${ }^{1}$, Sang Jin Kim ${ }^{1}$, Geun Woo Lee ${ }^{1,2}$, Kyung Jun Choi ${ }^{1}$ \\ ${ }^{1}$ Department of Ophthalmology, Samsung Medical Center, Sungkyunkwan University School of Medicine, Seoul, Korea \\ ${ }^{2}$ Department of Ophthalmology, Daegu Catholic University School of Medicine, Daegu, Korea
}

Purpose: This study aimed to establish and validate optical coherence tomography (OCT) based diagnostic criteria of high myopia.

Methods: This was a cross-sectional study including 100 eyes of high myopia with axial length larger than $26.5 \mathrm{~mm}$ and 100 control eyes, which were examined by spectral-domain OCT. Vertical and horizontal OCT of $9 \mathrm{~mm}$ scanning across fovea were analyzed. OCT characteristics including mirror artifact, scleral sink due to steep inclination of posterior sclera, scleral visibility, abnormality of outer retinal layer, foveoschisis, and dome-shaped macula were assessed in each group.

Results: The mean axial length was $28.65 \pm 2.07 \mathrm{~mm}$ (range, 26.51-34.59 mm) in high myopia group and $23.79 \pm 0.99 \mathrm{~mm}$ (range, 21.26-25.94 mm) in control group. Among the OCT characteristics noted at high frequency in the eyes with high myopia, three criteria achieving high sensitivity and specificity were determined: scleral sink over $500 \mu \mathrm{m}$, scleral visibility over $100 \mu \mathrm{m}$, and dome-shaped macula. Under conditions of presence of any of three criteria in either horizontal or vertical OCT scanning, the diagnostic sensitivity, specificity, positive predictive value, and negative predictive value for high myopia were found to be $95.0 \%, 98.0 \%, 98.0 \%$, and $95.1 \%$, respectively. In a new set of OCT images from 50 eyes with high myopia and 50 eyes of nonhigh myopia cases, the OCT-based criteria also proved similar level of diagnostic validity.

Conclusions: The OCT-based criteria, which directly addressed posterior scleral contour changes, may lead to an intuitive and accurate diagnosis of high myopia. Also, the criteria may contribute to early detection and monitoring of eyes that cannot be defined as high myopia but can progress. OCT may be useful for monitoring high myopia patients as OCT can detect myopia-associated retinal pathologies as well as scleral contour changes.

Key Words: Myopia, Optical coherence tomography, Sclera

Received: June 25, 2021 Final revision: November 12, 2021

Accepted: November 17, 2021

Corresponding Author: Se Woong Kang, MD, PhD. Department of Ophthalmology, Samsung Medical Center, Sungkyunkwan University School of Medicine, 81 Irwon-ro, Gangnam-gu, Seoul 06351, Korea. Tel: 82-23410-3562, Fax: 82-2-3410-0074, E-mail: swkang@skku.edu

This paper was presented in part at the 124th annual meeting of the Korean Ophthalmology Society, Seoul, Korea on November 1, 2020.
High myopia (HM) is one of the leading causes of blindness worldwide, especially in East Asia [1-4]. Although there is no single uniformly accepted definition, HM could be defined by the presence of a refractive error of at least -8.0 diopters or an axial length of $26.5 \mathrm{~mm}$ [5]. Pathologic signs have been reported by the presence of following changes: chorioretinal atrophy, myopic choroidal neovascularization, lacquer cracks, posterior staphyloma, myopic 
conus, and myopic optic neuropathy $[1,6,7]$. Recently, optical coherence tomography (OCT) has enabled us to describe new myopic disease entities such as myopic traction maculopathy and dome-shaped macula (DSM) [8,9]. Essentially, axial elongation is believed to play a key role in mediating these pathologic changes [10]. Histological stud- ies in HM eyes have provided evidence for scleral thinning at the posterior pole [11]. Progressive elongation and scleral thinning directly lead to changes in the curvature and radius of the sclera [11,12]. Consequent severe deformation of the sclera may be presented as posterior staphyloma. There have been reports which addressed scleral contour changes
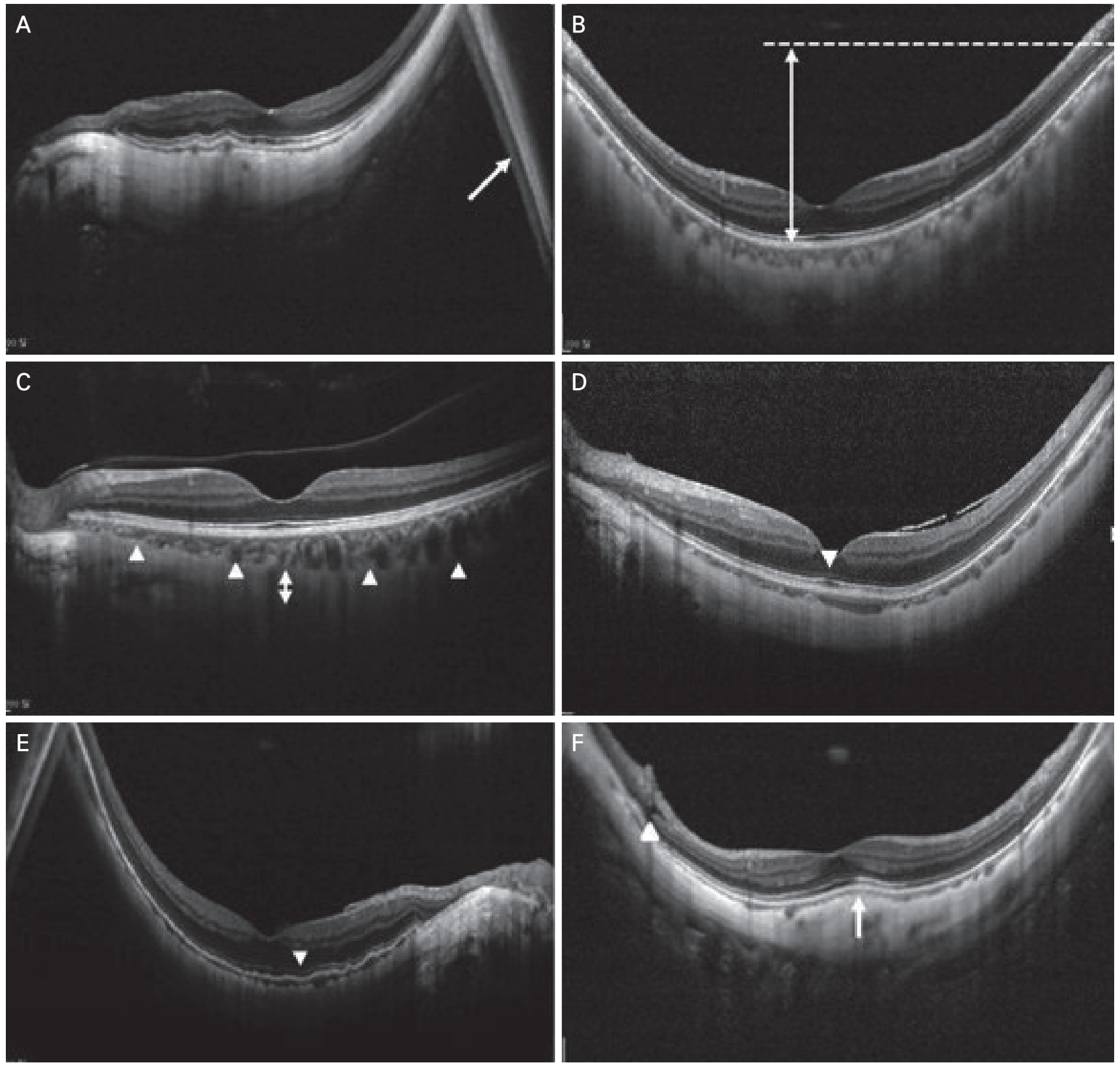

Fig. 1. Optical coherence tomography characteristics of high myopia. (A) Mirror artifact (arrow), image appears to be folded onto itself because of extreme structural curvature. (B) Large scleral sink (double arrow), a height difference in the retinal pigment epithelium due to steep inclination of sclera. (C) Enhanced scleral visibility (double arrow), arrowheads indicate a chorioscleral interface. (D) Disruption of interdigitation zone (arrowhead). (E) Low intensity or disruption of the ellipsoid zone (arrowhead). (F) Dome-shaped macula (arrow), relatively localized region of scleral thickening under the macula, arrowhead indicates paravascular inner retinal defect. 
in OCT as major characteristics of HM [10,12,13]. Although Fang et al. [14] reported OCT-based diagnosis in different stages of HM, their study mainly focused on choroidal thickness changes according to the different stages of myopic maculopathy. Few studies validated HM characteristics of OCT as the diagnostic criteria.

The advantage of OCT-based diagnosis is that the evaluation of posterior structures may reflect essential pathophysiology of HM [15]. Also, quantitative monitoring of the progression by OCT, which is much more frequently performed than axial length measurement, is feasible. It is useful especially in eyes with previous refractive surgery or cataract extraction [16,17]. It may also address the early phase of HM which cannot be defined by conventional criteria. This study was conducted to identify and validate novel OCT criteria for diagnosing HM.

\section{Materials and Methods}

The cross-sectional, case-control study was approved by the institutional review board of Samsung Medical Center and conformed to the Declaration of Helsinki. We retrospectively reviewed electronic medical records of 1,670
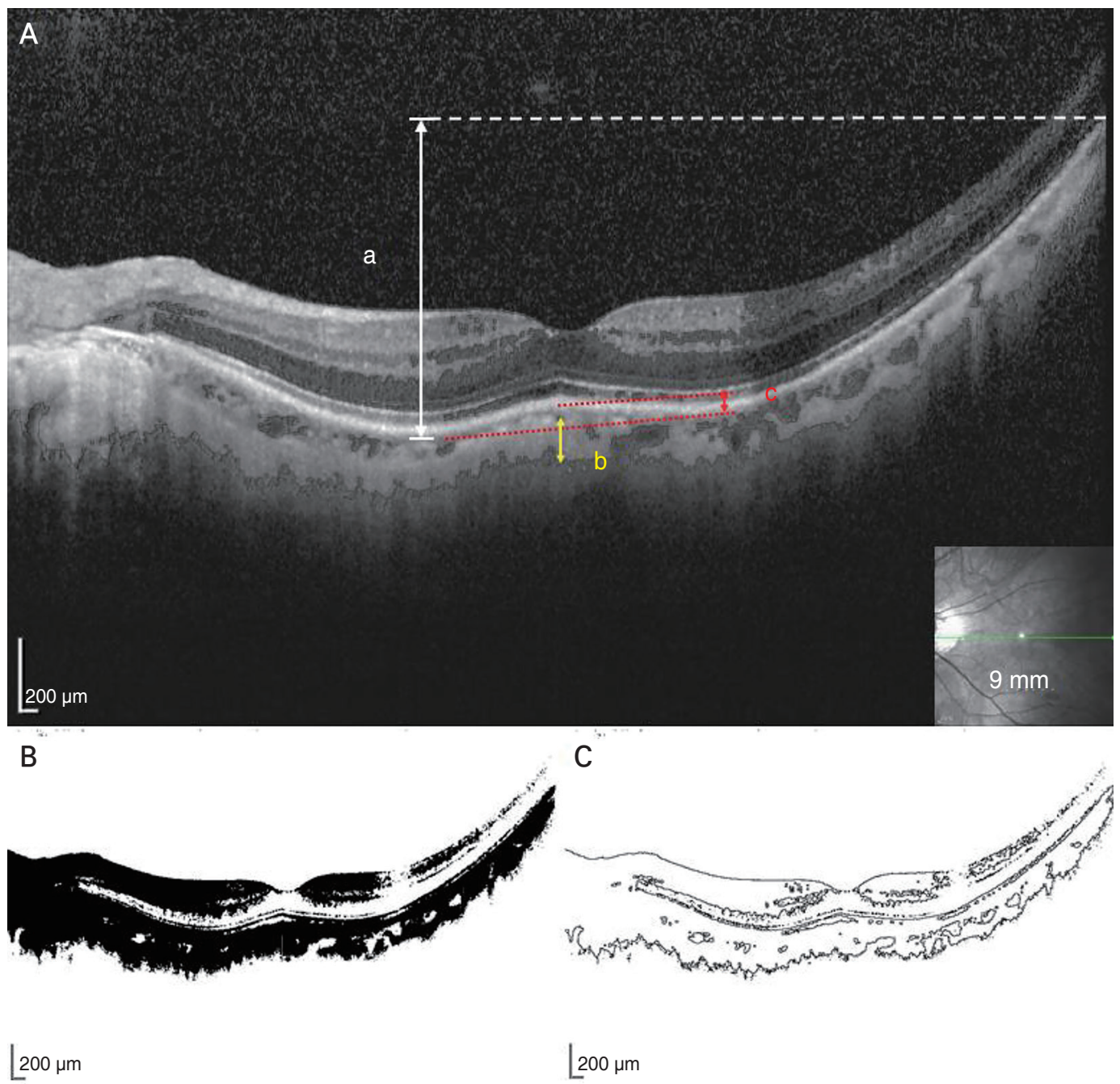

Fig. 2. Representative optical coherence tomography image of high myopia, describing the measurement of scleral sink, scleral visibility, and dome-shaped macula. (A) The $9 \mathrm{~mm}$ optical coherence tomography line scans through the fovea, the binarized and original images were overlapped for measurement. Scale bar indicates $200 \mu \mathrm{m}$; (a) indicates the amount of scleral sink, the height difference between the lowest and highest points of the retinal pigment epithelium; (b) indicates the distance of scleral visibility; and (c) indicates dome-shaped macula which is defined when inward protrusion of retinal pigment epithelial line is more than $50 \mu \mathrm{m}$ from the baseline. (B) The original image was converted to a binary image. (C) The binarized image was converted to a binarized outline image. 
eyes of 1,021 patients who had undergone preoperative examinations before cataract surgery in the Samsung Medical Center between June 1, 2018 and December 31, 2019. Among them, 100 eyes from 65 consecutive patients were enrolled for the HM group. As the refractive error was inaccurate due to cataract or previous refractive surgery, HM was defined as an axial length longer than $26.5 \mathrm{~mm}$ in this study. For the control group, 100 non-HM eyes from 65 cataract patients with age and sex-matched to HM group were enrolled for the same study period. Non-HM was defined as generally inconsistent with HM. In other words, patients with an axial length of less than $26.5 \mathrm{~mm}$ and without pathological myopic fundus features were selected for the control group. The exclusion criteria were a history of vitreoretinal surgery, poor quality OCT image due to severe cataract, and the presence of retinal diseases other than complications of HM.

All the patients underwent a complete ocular examination in preparation to undergo cataract surgery including color fundus photography or ultra-wide-field fundus photography (Optos, Dunfermline, UK), spectral-domain OCT (Spectralis HRA + OCT; Heidelberg Engineering, Heidelberg, Germany) with a wavelength of $870 \mathrm{~nm}$, and biometry based on swept-source OCT (Argos; Movu, Santa Clara, CA, USA) for the measurement of axial length.

\section{Analysis and measurement of spectral-domain OCT}

Horizontal and vertical OCT scans (9 $\mathrm{mm}$ in length) by enhanced depth imaging mode across the fovea were recorded. The scanning was conducted under a standardized imaging protocol and standardized mesopic lighting conditions. Each OCT B-scan had 25 to 35 frames averaged to improve the image quality. The horizontal and vertical scan were exported for the analysis of OCT characteristics including mirror artifact, scleral sink, disruption of interdigitation zone, disruption or low intensity of ellipsoid zone, peripapillary intrachoroidal cavitation, paravascular inner retinal defect, epiretinal membrane, choroidal neovascularization, foveoschisis, DSM, and macular hole (Fig. 1A-1F). These characteristics were independently evaluated by two examiners (YKS and GWL), both of whom were masked to HM or non-HM group. If there was a difference in the OCT analysis by the two examiners, we tried to consider a third-independent examiner (KJC).

All OCT images were analyzed using Image $\mathrm{J}$ software ver. 1.47 (US National Institutes of Health, Bethesda, MD, USA; http://imagej.nih.gov/ij/). In HM eyes, OCT frequently reveals steep inclination due to the high curvature of posterior sclera. It was expressed as "scleral sink" in this study. We defined the amount of scleral sink as the Y-axis difference between the lowest and highest points of retinal pigment epithelium in horizontal and vertical OCT scanning with a 9-mm scan length. If the mirror artifact was visible, the highest point of retinal pigment epithelium was measured as possible. The mirror artifact occurs when the area of interest to be imaged crosses the zero-delay line and results in an inverted image [18]. DSM was defined as an inward bulging of the retinal pigment epithelial line more than $50 \mu \mathrm{m}$ above a baseline (Fig. 2A-2C).

\section{Binarization of spectral-domain OCT images for the measurement of scleral visibility}

All exported spectral-domain OCT images were converted to binarization by Image $\mathrm{J}$ software. The binarization was done by a modified Niblack method. The binarized image was converted to outline binarized image for

Table 1. Baseline characteristics of high myopia group and control group

\begin{tabular}{lcc}
\hline Characteristics & High myopia group $(\mathrm{n}=65)$ & Control group $(\mathrm{n}=65)$ \\
\hline No. of eyes & 100 & 100 \\
Sex & & \\
$\quad$ Male & 36 & 35 \\
Female & 29 & 30 \\
Age (yr) & $50.2 \pm 13.4(11-79)$ & $50.1 \pm 13.2(13-79)$ \\
Axial length $(\mathrm{mm})$ & $28.65 \pm 2.07(26.51-34.59)$ & $23.79 \pm 0.99(21.26-25.94)$ \\
\hline
\end{tabular}

Values are presented as number or mean \pm standard deviation (range). 
objective measurement. We defined scleral visibility as the distance from the subfoveal choroid-scleral border to the lowest outline in the binarized outline image (Fig. 2). The length of scleral visibility in each eye was represented by the average of measurement in horizontal and vertical scanning across fovea.

\section{Determination and validation of OCT-based diagnostic criteria}

Statistical analysis was executed using SAS ver. 9.4 (SAS Institute, Cary, NC, USA) and R ver. 3.5.1 (The R Foundation for Statistical Computing, Vienna, Austria; https:// www.r-project.org/). The receiver operating characteristic analyses were performed to determine the optimal cutoff values of scleral sink and scleral visibility, which provide discrimination between the HM and control groups. The ideal cutoff value corresponded to the maximal value of the Youden index. The sensitivity, specificity, positive predictive value, and negative predictive value were evaluated for establishing putative diagnostic criteria. To validate the OCT-based diagnostic criteria of HM, we acquired a new set of OCT images from 50 eyes with HM and 50 eyes of non-HM cases. Based on the criteria, the independent ob-

A

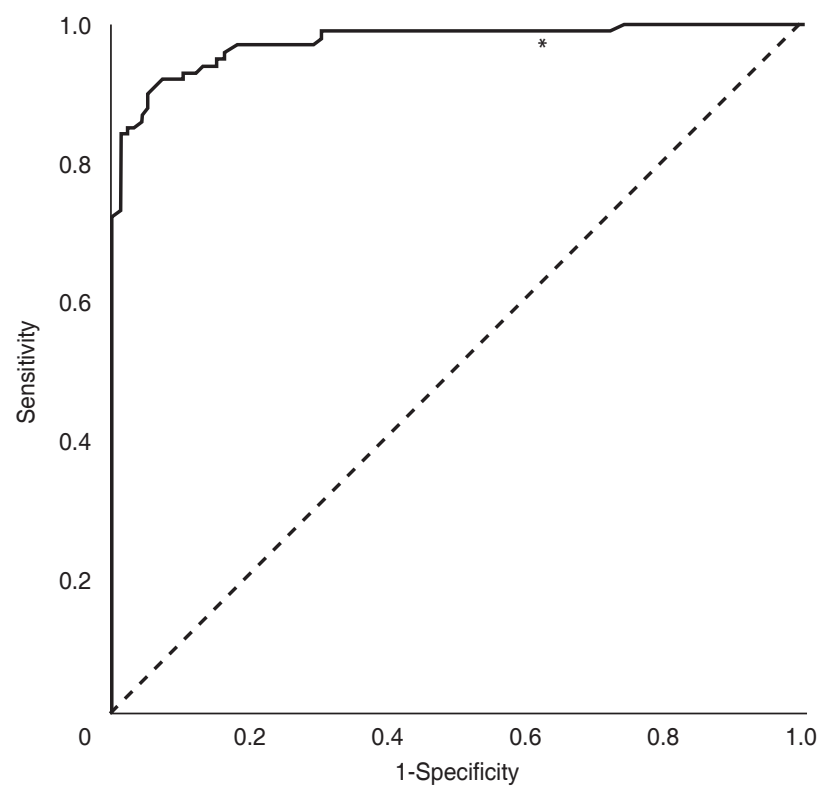

server blinded to the information of each case determined the diagnosis of HM among the new set of images.

\section{Results}

The clinical characteristics of the 100 eyes (65 patients) with HM were analyzed. There were 36 male patients and 29 female patients with a mean age of $50.2 \pm 13.4$ years (range, 11-79 years). The mean axial length was $28.65 \pm$ $2.07 \mathrm{~mm}$ (range, 26.51-34.59 mm). In age and sex-matched control eyes, 100 eyes (65 patients) were studied. There were 35 male patients and 30 female patients. The mean age was $50.1 \pm 13.1$ years (range, $13-79$ years) and the mean axial length was $23.79 \pm 0.99 \mathrm{~mm}(21.26-25.94 \mathrm{~mm})$ (Table 1). According to meta-analysis of pathologic myopia study classification [7], nine eyes had tessellated fundus, 73 eyes had diffuse chorioretinal atrophy, 14 eyes had patchy chorioretinal atrophy, and four eyes had macular atrophy. In the control group, no eyes showed HM fundus characteristics.

The analysis of OCT images in both groups revealed the following results. Mirror artifact was noted only in $41 \mathrm{HM}$ eyes. In the control group, the mean amount of scleral sink

B

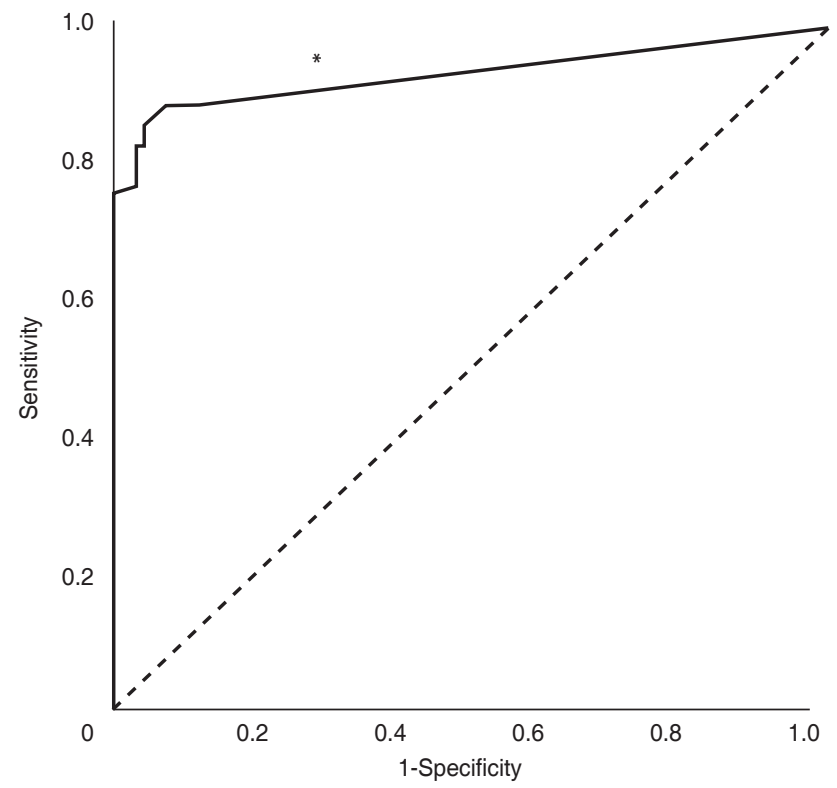

Fig. 3. Receiver operator characteristic (ROC) curve for (A) scleral sink and (B) scleral visibility. The area under the curve of the ROC curve obtained from the scleral sink is 0.975 and the $p$-value is $<0.001$. The cutoff value obtained through the Youden index was $402 \mu \mathrm{m}$ (asterisk, 91\% sensitivity, 94\% specificity). The area under the curve of the ROC curve obtained from scleral visibility was 0.929 and the $p$-value was $<0.001$. The cutoff value obtained through the Youden index was $32 \mu \mathrm{m}$ (asterisk, $86.5 \%$ sensitivity, $94.5 \%$ specificity). 


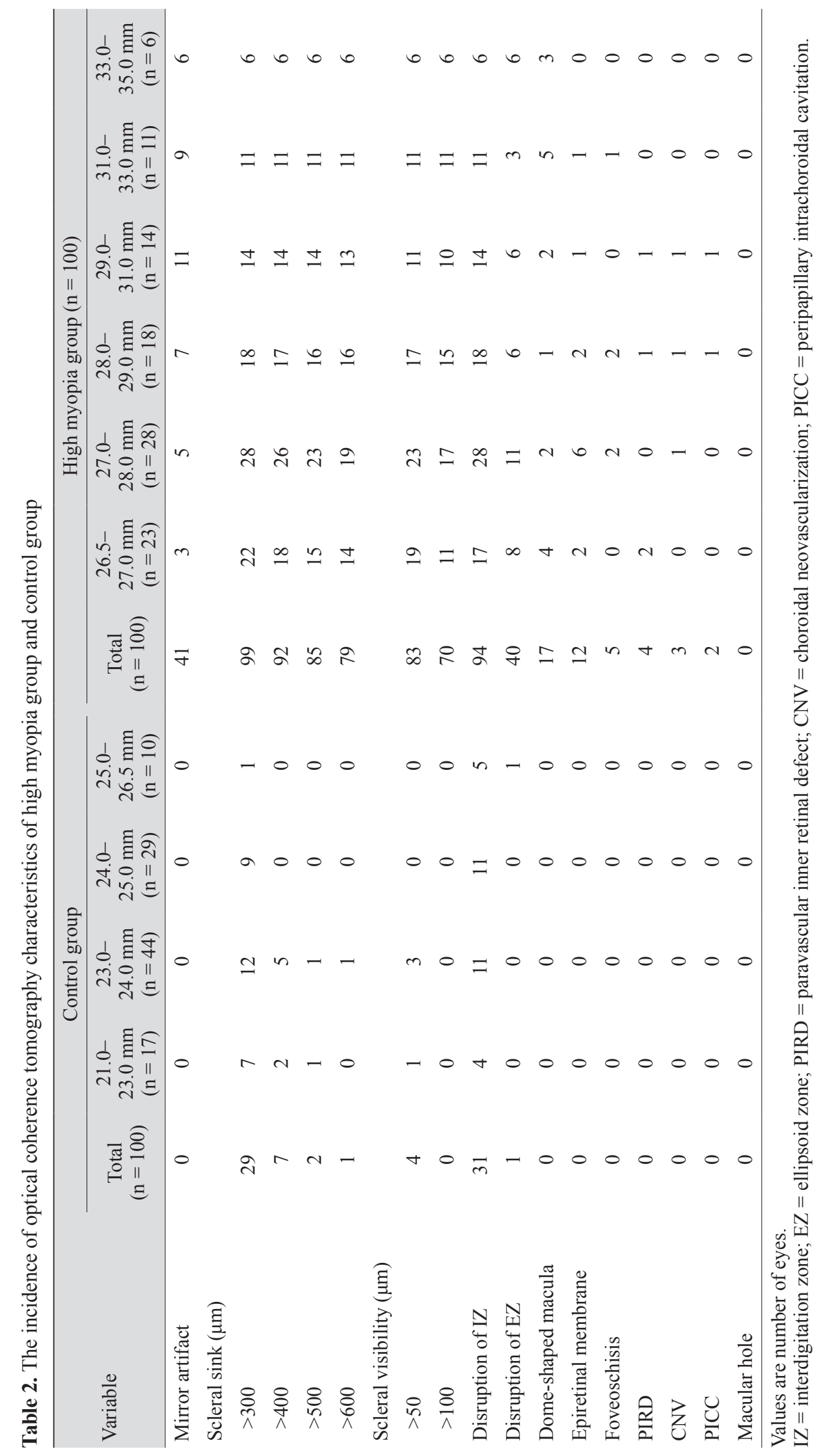


was measured as 195.8.9 $\pm 91.4 \mu \mathrm{m}$ in the horizontal direction and $221.8 \pm 112.7 \mu \mathrm{m}$ in the vertical direction. In $\mathrm{HM}$ group, the mean amount of scleral sink was $897.0 \pm 462.4 \mu \mathrm{m}$ in the horizontal direction and $925.9 \pm 406.0 \mu \mathrm{m}$ in the vertical direction, which were larger than those in the control group $(p<0.001)$. The scleral layer was usually invisible in the control group. The length of subfoveal scleral visibility in the binarized outline image was measured on both horizontal and vertical scans and was averaged. The mean scleral visibility was $4.7 \pm 15.9 \mu \mathrm{m}$ in the control group and $142.1 \pm 89.6 \mu \mathrm{m}$ in HM group $(p<0.001)$. In 100 eyes of HM group, the OCT revealed DSM in 17 eyes, epiretinal membrane in 12 eyes, foveoschisis in five eyes, paravascular inner retinal defect in four eyes, choroidal neovascularization in three eyes, and peripapillary intrachoroidal cavitation in two eyes. Disruption of the interdigitation zone was observed in 94 eyes of the HM group and 31 eyes of the control group. Low intensity or disruption of the ellipsoid zone was observed in 40 eyes of the HM group and 1 eye of the control group. The macular hole was not observed in all eyes. Receiver operating characteristic analyses were performed to determine the optimal value that can discriminate HM and control eyes only by OCT. The cutoff value that measured the amount of scleral sink was $402 \mu \mathrm{m}$, with $91 \%$ sensitivity and $94 \%$ specificity. The cutoff value of scleral visibility was $32 \mu \mathrm{m}$, with $86.5 \%$ sensitivity and $94.5 \%$ specificity (Fig. 3A, 3B).

Besides, for a more intuitive judgment, we analyzed the number of eyes with the amount of scleral sink over 300 , 400,500 , and $600 \mu \mathrm{m}(1.5,2,2.5,3$ times of reference; $200 \mu \mathrm{m}$ scale bar) and the length of scleral visibility over 50 and $100 \mu \mathrm{m}$ (quarter, half of the scale bar). The amount of scleral sink over $300,400,500$, and $600 \mu \mathrm{m}$ was observed in $99,92,85$, and 79 eyes, respectively, among 100 eyes of $\mathrm{HM}$, and in 29, 7, 2, and 1 eye, respectively, among 100 control eyes. The scleral visibility over 50 and $100 \mu \mathrm{m}$ was observed in 83 and 70 eyes of the HM group, respectively, and in 4 and 0 eyes of the control group, respectively. The detailed incidence of OCT characteristics in HM and nonHM control groups according to the axial length is shown in Table 2.

To establish the OCT-based diagnostic criteria of HM, we selected the combination of parameters enabling high sensitivity and specificity of diagnosis with the least number of parameters. Based on the dataset from 200 eyes in the current study, the combination of the following three parameters was identified to satisfy the above requirement. The three parameters were the amount of scleral sink over $500 \mu \mathrm{m}$, the length of scleral visibility over $100 \mu \mathrm{m}$, and DSM. The presence of any of the three parameters in the horizontal or vertical OCT scanning served as diagnostic criteria for HM with diagnostic sensitivity, specificity, positive predictive value, and negative predictive value as $95.0 \%, 98.0 \%, 98.0 \%$, and $95.1 \%$, respectively (Table 3). In a new set of horizontal and vertical OCT images from 50 eyes with HM and 50 eyes of non-HM cases, the OCTbased diagnostic criteria of HM also revealed high sensitivity, specificity, positive predictive value, and negative predictive value as $100 \%, 96.0 \%, 96.2 \%$, and $100 \%$, respectively (Table 3).

\section{Discussion}

Although the importance of myopia is increasing, the definition of myopia has not been consistent $[19,20]$. Recently, the diagnosis of high myopia by evaluating the refractive error has become increasingly difficult because of cataract progression and prior refractive or cataract surgery. And in this study, nine eyes had only tessellated fundus and were unable to diagnose pathologic myopia according to the meta-analyses of pathologic myopia study

Table 3. Sensitivity, specificity, and predictive value of OCT-based diagnostic criteria in detecting high myopia

\begin{tabular}{lcccc}
\hline The presence of any one of three OCT $^{*}$ characteristics & $\begin{array}{c}\text { Sensitivity } \\
(\%)\end{array}$ & $\begin{array}{c}\text { Specificity } \\
(\%)\end{array}$ & $\begin{array}{c}\text { Positive predictive } \\
\text { value }(\%)\end{array}$ & $\begin{array}{c}\text { Negative predictive } \\
\text { value }(\%)\end{array}$ \\
\hline $\begin{array}{c}\text { Scleral sink }>500 \mu \mathrm{m} \text { in horizontal or vertical direction, } \\
\text { scleral visibility }>100 \mu \mathrm{m} \text {, or dome-shaped macula }\end{array}$ & 95.0 & 98.0 & 98.0 & 95.1 \\
$\begin{array}{l}\text { Validation dataset } \\
\text { Validat }\end{array}$ & 100 & 96.0 & 96.2 & 100 \\
\hline
\end{tabular}

OCT $=$ optical coherence tomography.

*Spectral-domain OCT with $9 \mathrm{~mm}$ scan length, $870 \mathrm{~nm}$ scanning laser; ${ }^{\dagger}$ Validation dataset consisted of 50 eyes with pathologic myopia and 50 eyes with control. 
classification (category 1). Recently, The OCT examinations are much more frequently conducted than axial length measurements. Because OCT has become an essential test for ophthalmology by providing quantitative and qualitative assessments [21], OCT-based diagnosis of HM is hypothesized to be of benefit.

In HM, OCT has enabled the detailed observation of macular pathologies with the progression of axial elongation and stretching of the globe, even in the presence of media opacity such as cataract $[19,22]$. Several OCT characteristics such as paravascular inner retinal defect, peripapillary intrachoroidal cavitation, paravascular retinal cysts, vascular microfolds, paravascular lamellar holes, epiretinal membrane, vitreomacular traction, incomplete posterior vitreous detachment, myopic tractional maculopathy, tractional internal limiting membrane detachment, myopic foveoschisis, choroidal neovascular membrane, posterior retinal detachment, and macular hole were reported $[15,23,24]$. The present study revealed that certain OCT characteristics such as mirror artifact, high amount of scleral sink, large length of scleral visibility, paravascular inner retinal defect, epiretinal membrane, choroidal neovascularization, foveoschisis, and DSM were not observed at all in non-HM control eyes.

Although the longer scanning length OCT and ultra-wide field OCT is recently introduced, the $9 \mathrm{~mm}$ OCT scanning length has been widely used with successful visualization of the shape of the posterior pole [13]. In this study, we investigated the morphologic characteristics of $\mathrm{HM}$ in terms of posterior chorioscleral configurations via OCT. The posterior chorioscleral structural alterations related to HM resulted in steep inclinations on OCT [13], which was expressed as a scleral sink in this study. In the control group, scleral sink over $500 \mu \mathrm{m}$ was noted only in $2 \%$ of eyes. The result signifies that scleral sink over 500 $\mu \mathrm{m}$ confirms the diagnosis of the eye as HM with $98 \%$ specificity. Scleral sink over $500 \mu \mathrm{m}$ was used as the diagnostic criteria using OCT while considering specificity, sensitivity, and cutoff values.

OCT in HM eyes with significant thinning of the retinochoroidal layers may allow visualization of the whole choroidal layer, posterior sclera, episclera, and in some cases, even Tenon's capsule [25]. Recently, Fang et al. [14] reported that progressive and continuous choroidal thinning plays a key role in the progression from no maculopathy to tessellation and to diffuse atrophy. However, the resolution of the OCT was insufficient to reveal the entire posterior scleral layer, except for severe myopia [26]. Because of these limitations associated with the current OCT technology, the report on the posterior scleral layer is rare except on DSM [26]. In this study, we developed a technique of binarization by Image J, to quantify the visibility of posterior sclera. In the non-HM group, the sclera was invisible in $89 \%$ of eyes and the maximum scleral visibility was $80 \mu \mathrm{m}$, thereby signifying that the scanning light cannot penetrate enough into sclera in non-HM control eyes as they have intact retinal pigment epithelium and choroid than HM eyes. Thus, the scleral visibility over $100 \mu \mathrm{m}$ constitutes one of the diagnostic criteria for $\mathrm{HM}$ with $70 \%$ sensitivity and $100 \%$ specificity. We utilized SD-OCT with a wavelength of $870 \mathrm{~nm}$. In case of employment of OCT utilizing longer wavelength, such as swept-source OCT, the cutoff value might be longer than $100 \mu \mathrm{m}$. Also, age and skin color are correlated with melanin pigmentation in retinal pigment epithelium and choroid, which would influence the amount of scleral visibility.

It has been reported that DSM was observed in $9.3 \%$ to $20.1 \%$ of highly myopic eyes [27-29]. In this study, DSM over $50 \mu \mathrm{m}$ of dome height was noted in $17.0 \%$ of the HM group, similar to previous studies. DSM was not observed in the control group. Consequently, the presence of DSM also constitutes one of the diagnostic criteria for HM with $100 \%$ specificity and $17 \%$ sensitivity. Although the underlying mechanism for the development of DSM remains unclear, the occurrence of DSM was potentially related to the local thickening of the subfoveal sclera and the parafoveal scleral thinning in a progressive and symmetric manner $[26,30,31]$.

Several reports suggest that the disruption or decreased reflectivity of the ellipsoid zone is one of the OCT characteristics of HM [15,32]. The loss of the interdigitation zone was also thought to be the evidence of overlying photoreceptor degeneration [33,34]. Circumscribed loss of outer foveal layer is observed in various diseases such as geographic atrophy and choroideremia [35]. However, in HM eyes, diffuse loss of outer foveal layer is observed. The current study also revealed that intact four hyperreflective bands of the outer foveal layer were not delineated in high proportion in HM group. Other OCT features such as epiretinal membrane, choroidal neovascularization, foveoschisis, and paravascular inner retinal defect were observed in HM but not in control eyes. Although the OCT 
characteristics corresponding to degeneration in the outer foveal layer are of great importance, the changes at the cellular level are rather frequently noted in diverse maculopathies such as age-related macular degeneration and pachychoroid spectrum diseases [33,36]. Thus, microstructural change such as disruption in the ellipsoid zone and interdigitation zone was not considered as a diagnostic criterion. OCT-based diagnostic criteria were selected in consideration of the gross structural changes reflecting the progressive increase in axial length. Diagnostic criteria were selected among combinations of several OCT characteristics, enabling the highest diagnostic sensitivity and specificity of HM with the least number of characteristics. Based on this rule, scleral sink, scleral visibility, and DSM were determined as diagnostic criteria (Table 3). The diagnostic value of the OCT-based criteria was also supported by the validation test utilizing a new set of 100 cases.

There are several limitations to this study. First, our study population only consisted of Asian ethnicity. Retinochoroidal thickness and the amount of pigmentation may vary significantly in other ethnicities [37,38]. A possible reason for invisible sclera in most of the non-HM eyes would be the presence of greater pigmentation in Asian eyes, compared to Caucasians. Further studies in other ethnic populations would be meaningful. Second, our study only described measurements and characteristics using the OCT with a wavelength of $870 \mathrm{~nm}$. Different OCT modalities have different resolution and speed; however, most of the features described in this study might be observed on other OCT modalities. Third, because the inclusion criteria for HM group depended only on axial length over $26.5 \mathrm{~mm}$, the HM group in the current study may not represent all the HM eyes. For example, a case with a refractive error larger than -8 diopters, but an axial length of less than $26.5 \mathrm{~mm}$ was not included in this study. However, such a case might not be so frequent in the real world.

In conclusion, we propose OCT-based diagnostic criteria of HM in this study. With high sensitivity and specificity, we may make the diagnosis of $\mathrm{HM}$ in the eyes with any of three OCT characteristics; scleral sink over $500 \mu \mathrm{m}$ in the horizontal or vertical direction, or scleral visibility over $100 \mu \mathrm{m}$, or DSM. This novel OCT criteria that focus on scleral contour can be meaningful for diagnosing HM patients who had unknown or inaccurate refractive error due to cataract progression, previous cataract or refractive surgery.
HM is defined as refractive error or axial length. However, considering that OCT examination is conducted much more frequently than axial length test, that the early phase of HM is not obvious by conventional diagnostic criteria, and that HM is a progressive disease, OCT-based suspicion may be meaningful for eyes that cannot be defined as HM by conventional criteria to monitoring and early detection.

Conflicts of Interest: None.

Acknowledgements: None.

Funding: None.

\section{References}

1. Morgan IG, Ohno-Matsui K, Saw SM. Myopia. Lancet 2012;379:1739-48.

2. Iwase A, Araie M, Tomidokoro A, et al. Prevalence and causes of low vision and blindness in a Japanese adult population: the Tajimi Study. Ophthalmology 2006;113:135462.

3. Varma R, Kim JS, Burkemper BS, et al. Prevalence and causes of visual impairment and blindness in Chinese American adults: the Chinese American Eye Study. JAMA Ophthalmol 2016;134:785-93.

4. Wong TY, Ferreira A, Hughes R, et al. Epidemiology and disease burden of pathologic myopia and myopic choroidal neovascularization: an evidence-based systematic review. Am J Ophthalmol 2014;157:9-25.

5. Tokoro T. On the definition of pathologic myopia in group studies. Acta Ophthalmol Suppl 1988;185:107-8.

6. Ikuno Y, Ohno-Matsui K, Wong TY, et al. Intravitreal aflibercept injection in patients with myopic choroidal neovascularization: the MYRROR Study. Ophthalmology 2015;122:1220-7.

7. Ohno-Matsui K, Kawasaki R, Jonas JB, et al. International photographic classification and grading system for myopic maculopathy. Am J Ophthalmol 2015;159:877-83.

8. Panozzo G, Mercanti A. Optical coherence tomography findings in myopic traction maculopathy. Arch Ophthalmol 2004;122:1455-60.

9. Gaucher D, Erginay A, Lecleire-Collet A, et al. Domeshaped macula in eyes with myopic posterior staphyloma. Am J Ophthalmol 2008;145:909-14.

10. Moriyama M, Ohno-Matsui K, Hayashi K, et al. Topo- 
graphic analyses of shape of eyes with pathologic myopia by high-resolution three-dimensional magnetic resonance imaging. Ophthalmology 2011;118:1626-37.

11. McBrien NA, Gentle A. Role of the sclera in the development and pathological complications of myopia. Prog Retin Eye Res 2003;22:307-38.

12. Ohno-Matsui K, Akiba M, Modegi T, et al. Association between shape of sclera and myopic retinochoroidal lesions in patients with pathologic myopia. Invest Ophthalmol Vis Sci 2012;53:6046-61.

13. Miyake M, Yamashiro K, Akagi-Kurashige Y, et al. Analysis of fundus shape in highly myopic eyes by using curvature maps constructed from optical coherence tomography. PLoS One 2014;9:e107923.

14. Fang Y, Du R, Nagaoka N, et al. OCT-based diagnostic criteria for different stages of myopic maculopathy. Ophthalmology 2019;126:1018-32.

15. You QS, Peng XY, Xu L, et al. Myopic maculopathy imaged by optical coherence tomography: the Beijing eye study. Ophthalmology 2014;121:220-4.

16. Kezirian GM, Parkhurst GD, Brinton JP, Norden RA. Prevalence of laser vision correction in ophthalmologists who perform refractive surgery. J Cataract Refract Surg 2015;41:1826-32.

17. Erie JC. Rising cataract surgery rates: demand and supply. Ophthalmology 2014;121:2-4.

18. Ho J, Castro DP, Castro LC, et al. Clinical assessment of mirror artifacts in spectral-domain optical coherence tomography. Invest Ophthalmol Vis Sci 2010;51:3714-20.

19. Ohno-Matsui K, Lai TY, Lai CC, Cheung CM. Updates of pathologic myopia. Prog Retin Eye Res 2016;52:156-87.

20. Flitcroft DI, He M, Jonas JB, et al. IMI: defining and classifying myopia. A proposed set of standards for clinical and epidemiologic studies. Invest Ophthalmol Vis Sci 2019;60: M20-30.

21. Wang SK, Guo X, Xiao O, et al. Qualitative and quantitative assessment of posterior segment optical coherence tomography images using standard photos: the Liwan Eye Study. BMJ Open 2017;7:e017923.

22. McKeague M, Sharma P, Ho AC. Evaluation of the macula prior to cataract surgery. Curr Opin Ophthalmol 2018;29:4-8.

23. Ng DS, Cheung CY, Luk FO, et al. Advances of optical coherence tomography in myopia and pathologic myopia. Eye (Lond) 2016;30:901-16.

24. Faghihi H, Hajizadeh F, Riazi-Esfahani M. Optical coherence tomographic findings in highly myopic eyes. $\mathrm{J} \mathrm{Oph-}$ thalmic Vis Res 2010;5:110-21.

25. Ohno-Matsui K, Fang Y, Morohoshi K, Jonas JB. Optical coherence tomographic imaging of posterior episclera and Tenon's capsule. Invest Ophthalmol Vis Sci 2017;58:3389-94.

26. Imamura Y, Iida T, Maruko I, et al. Enhanced depth imaging optical coherence tomography of the sclera in domeshaped macula. Am J Ophthalmol 2011;151:297-302.

27. Ohsugi H, Ikuno Y, Oshima K, et al. Morphologic characteristics of macular complications of a dome-shaped macula determined by swept-source optical coherence tomography. Am J Ophthalmol 2014;158:162-70.

28. Liang IC, Shimada N, Tanaka Y, et al. Comparison of clinical features in highly myopic eyes with and without a dome-shaped macula. Ophthalmology 2015;122:1591-600.

29. Ceklic L, Wolf-Schnurrbusch U, Gekkieva M, Wolf S. Visual acuity outcome in RADIANCE study patients with dome-shaped macular features. Ophthalmology 2014;121: 2288-9.

30. Dai F, Li S, Wang Y, et al. Correlation between posterior staphyloma and dome-shaped macula in high myopic eyes. Retina 2020;40:2119-26.

31. Ellabban AA, Tsujikawa A, Muraoka Y, et al. Domeshaped macular configuration: longitudinal changes in the sclera and choroid by swept-source optical coherence tomography over two years. Am J Ophthalmol 2014;158:106270.

32. Wang NK, Wu YM, Wang JP, et al. Clinical characteristics of posterior staphylomas in myopic eyes with axial length shorter than 26.5 millimeters. Am J Ophthalmol 2016;162: 180-90.

33. Sadda SR, Guymer R, Holz FG, et al. Consensus definition for atrophy associated with age-related macular degeneration on OCT: classification of atrophy report 3. Ophthalmology 2018;125:537-48.

34. AttaAllah HR, Omar I, Abdelhalim AS. Assessment of posterior segment using spectral domain OCT in highly myopic eyes. Open Ophthalmol J 2017;11:334-45.

35. Goldberg NR, Greenberg JP, Laud K, et al. Outer retinal tubulation in degenerative retinal disorders. Retina 2013;33:1871-6.

36. Fujita A, Aoyama Y, Tsuneyoshi S, et al. Association between visual function and the integrity of residual ellipsoid zone in resolved central serous chorioretinopathy. Sci Rep 2019;9:12433.

37. Wolf-Schnurrbusch UE, Roosli N, Weyermann E, et al. Ethnic differences in macular pigment density and distri- 
bution. Invest Ophthalmol Vis Sci 2007;48:3783-7.

38. Bafiq R, Mathew R, Pearce E, et al. Age, sex, and ethnic variations in inner and outer retinal and choroidal thickness on spectral-domain optical coherence tomography. Am $J$ Ophthalmol 2015;160:1034-43. 